\title{
Higher mean arterial pressure increases risk of in-hospital mortality in aneurysmal subarachnoid hemorrhage
}

\author{
Nadya Noor Ramadhania*,Ahmad Faisal Darmawan*, and Achmad Firdaus Sani*
}

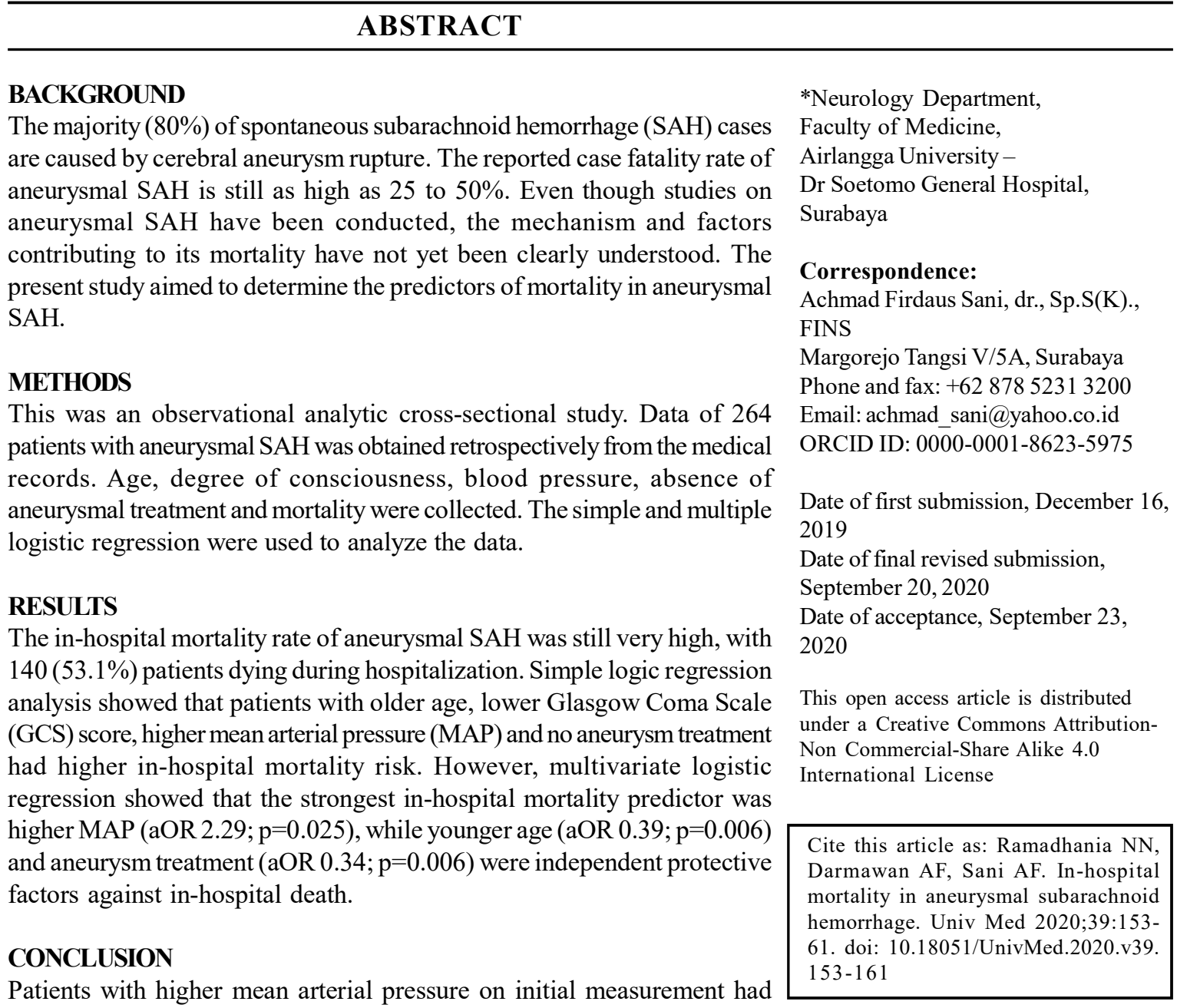
higher risks of mortality. More endovascular neurointervention facilities are needed to decrease the mortality rate of aneurysmal SAH.

Keywords : Cerebral aneurysm, mortality, subarachnoid hemorrhage, stroke 


\section{INTRODUCTION}

Subarachnoid hemorrhage (SAH) is a form of bleeding that occurs in the subarachnoid space between the pia mater and arachnoid mater, whereas its most frequent cause is from traumatic injury. Even so, spontaneous SAH is also commonly found as one of the forms of stroke. ${ }^{(1)}$ The reported incidence rate of SAH varies from 6.2 in Finland, ${ }^{(2)}$ Nigeria $6.51,{ }^{(3)} \mathrm{US}$ $7.7^{\text {(4) }}$ per 100,000 population each year and Japan ${ }^{(5)} 27.63$ per 100,000 person-years, with women still predominating, namely $71.9 \%$ in Kenya ${ }^{(6)}$ and $61.6 \%$ in China. ${ }^{(7)}$

The majority of spontaneous SAH is caused by ruptured cerebral aneurysm, which accounts for 70 to $80 \%$ of cases. ${ }^{(8)}$ Cerebral aneurysm occurs in 1 to $3 \%$ of the population. ${ }^{9)}$ The detection rate of unruptured cerebral aneurysm worldwide has been growing due to advancement of CT and MR angiographies and its treatment has been widely discussed to prevent future aneurysmal SAH (aSAH). ${ }^{(8)}$ Some countries have even started screening of first-degree relatives of aSAH patients. ${ }^{(10,11)}$ This has not yet been carried out in Indonesia, because the use of CT scan and MRI in nonsymptomatic patients is still not routinely done. Prior studies regarding predictors of mortality and poor outcome in aSAH have been done. Aneurysm re-bleeding and delayed cerebral ischemia in the course of this disease have been the strongest independent predictors of unfavorable outcome. ${ }^{(12,13)}$ Higher age was found to be associated with death and worse outcomes in various studies. ${ }^{(14-16)}$ Patients having in-hospital complications such as nosocomial infection and pneumonia experienced significantly worse outcomes. ${ }^{(17-19)}$ Radiological findings including the characteristics of aneurysms ${ }^{(14,19)}$ and other space-occupying lesions on CT-scan ${ }^{(14,20)}$ and laboratory findings such as red cell distribution width (RDW) ${ }^{(21)}$ and leukocyte counts ${ }^{(22)}$ were also found to be associated with patients' outcome. Witsch et al. ${ }^{(16)}$ and Ahn et al. ${ }^{(23)}$ have even developed scores that are used to predict patient outcome. ${ }^{(16,23)}$

An understanding of the pathophysiology of aSAH and evidence-based recommendations concerning its acute management might have important roles in decreasing aSAH mortality rates; ${ }^{(24,25)}$ however, it remains unclear whether this decrease in mortality can be attributed to a specific cause. The key to further improving survival after aSAH is to identify and eliminate factors or events that predict a negative outcome. This study aimed to identify the factors associated with in-hospital mortality in aSAH patients.

\section{METHODS}

\section{Research design}

This was an observational analytic crosssectional study conducted in Dr. Soetomo General Hospital, Surabaya, from March 2013 to July 2018.

\section{Research subjects}

The population in this study consisted of patients with SAH hemorrhage who had been admitted to Dr. Soetomo General Hospital, Surabaya. The inclusion criterion was patients with ICD-10 code for non-traumatic SAH (I60.9). Exclusion criteria were patients with known arteriovenous malformation, those with incomplete medical records and those with missing data in recorded initial clinical assessments and history-taking. A total sample of 264 subjects was used in this study. Subjects were then divided into two groups, survivors and non-survivors.

\section{Measurements}

Data were all obtained from subjects' medical records. We defined SAH as patients with symptoms of acute onset of thunderclap headache, seizures, focal neurologic signs, or decrease of consciousness that showed subarachnoid high attenuation on CT-scan. Patients' ages were taken from their national 
ID card. Degree of consciousness in Glasgow Coma Scale (GCS) and blood pressure were measured in the emergency department during admission to our hospital. History of hypertension, diabetes mellitus, cerebrovascular diseases and smoking were taken by asking the patients and their families. Aneurysm treatment data was obtained from the procedure report in the medical record.

The independent variables were all dichotomous, patients' age was categorized into $\geq 45$ years and $<45$ years. We used $135 \mathrm{mmHg}$ as the cutoff point for the mean arterial pressure (MAP). Patients' degree of consciousness that was scored in the Glasgow Coma Scale (GCS) was divided into $\geq 13$ and $<13$. The other variables were categorized into present (yes) and absent (no) according to history-taking and medical records.

\section{Statistical analysis}

Simple logistic regression analysis was used to analyze the relationship between dependent and independent variables. The results of the analysis were expressed as Odds Ratio (OR), 95\% Confidence Interval (CI) and p-value. Significant results are the ones with a p-value of less than 0.05 .

The independent variables that had $\mathrm{p}<0.25$ in the bivariate analysis were included in the multivariate logistic regression analysis. The results were expressed as Adjusted Odds Ratio (aOR), 95\% Confidence Interval (CI) and $\mathrm{p}$ value, which was considered significant if $\mathrm{p}$ value $<0.05$.

\section{Ethical clearance}

The ethical committee of Dr. Soetomo General Hospital issued ethical clearance no. 1573/KEPK/X/2019 on October $9^{\text {th }}, 2019$.

\section{RESULTS}

Subjects in this study were divided into two groups, those who survived and those who died during hospitalization. Hundred and forty patients (53.1\%) did not survive hospital treatment. The median age of the subjects was 55 years (interquartile range [IQR] 47-63), with initial mean arterial pressure of $116.45 \pm 22.69 \mathrm{mmHg}$. Degree of consciousness was measured with GCS Score, with median of 11 (IQR 9-15). More than half (57.2\%) of the subjects had a history of hypertension, and most of the subjects did not have a history of diabetes mellitus ( $88.3 \%)$, cerebrovascular diseases (89.4\%), and smoking (90.9\%). During hospitalization, aneurysm treatment was only administered to 39 patients $(14.8 \%)$, in which 36 of them underwent endovascular coiling, two patients underwent surgical clipping and one patient underwent both procedures (data not shown).

Simple logistic regression analysis showed that the risk of mortality was lower in younger patients than in the older ones $(\mathrm{OR}=0.36 ; 95 \%$ $\mathrm{CI}=0.19-0.69 ; \mathrm{p}=0.002)$. Similar finding was found in patients with higher GCS score during admission $(\mathrm{OR}=0.42 ; 95 \% \mathrm{CI}=0.25-0.71$; $\mathrm{p}=0.001)$. Patients with higher initial MAP had 2.82 times higher risk of death $(\mathrm{OR}=2.82$; $\mathrm{CI}$ 95\% 1.44-5.52; $\mathrm{p}=0.002)$. As expected, aneurysm treatment was found to be a protective factor against mortality $(\mathrm{OR}=0.27 ; 95 \% \mathrm{CI}=$ $0.13-0.58 ; \mathrm{p}<0.001)$. History of hypertension, diabetes mellitus, smoking and cerebrovascular disease were not found to be associated with mortality in aSAH (Table 1).

Multivariate analysis found that age, MAP, and aneurysm treatment were the factors associated with mortality in aSAH. Younger subjects had a smaller risk of death (aOR 0.39; CI95\% 0.20-0.76; $\mathrm{p}=0.006$ ), those with higher mean arterial pressure had a higher risk of death (aOR 2.29; CI 95\% 1.10-4.39; $\mathrm{p}=0.025)$ and the ones who underwent aneurysm treatment were more likely to survive (aOR 0.34; CI 955 0.160.74; $p=0.006$ ) (Table 2). Despite its significance, this logistic regression model had area under ROC of 0.63 (CI 95\% 0.57-0.68) (Figure 1). 
Table 1. Relationship between clinical predictors and in-hospital mortality,

estimated using simple logistic regression analysis

\begin{tabular}{|c|c|c|c|c|c|c|c|}
\hline & \multicolumn{2}{|c|}{ Non-survivors $(n=140)$} & \multicolumn{2}{|c|}{ Survivors $(\mathrm{n}=124)$} & \multirow{2}{*}{ OR } & \multirow{2}{*}{ 95\% CI } & \multirow{2}{*}{ p-value } \\
\hline & $\mathbf{n}$ & $\%$ & $\mathbf{n}$ & $\%$ & & & \\
\hline Age & & & & & 0.36 & $0.19-0.69$ & 0.002 \\
\hline$<45$ years & 17 & 12.2 & 34 & 117.5 & & & \\
\hline$\geq 45$ years & 123 & 87.8 & 90 & 72.5 & & & \\
\hline GCS & & & & & 0.42 & $0.25-0.71$ & 0.001 \\
\hline $13-15$ & 45 & 35.1 & 64 & 54.7 & & & \\
\hline$\leq 12$ & 87 & 64.9 & 53 & 45.3 & & & \\
\hline MAP & & & & & 2.82 & $1.44-5.52$ & 0.002 \\
\hline$\geq 135 \mathrm{mmHg}$ & 37 & 26.4 & 14 & 11.3 & & & \\
\hline$<135 \mathrm{mmHg}$ & 103 & 73.6 & 110 & 88.7 & & & \\
\hline Hypertension & & & & & 1.12 & $0.69-1.83$ & 0.631 \\
\hline Yes & 82 & 58.6 & 69 & 55.7 & & & \\
\hline No & 58 & 41.4 & 55 & 44.3 & & & \\
\hline Diabetes mellitus & & & & & 1.08 & $0.51-2.31$ & 0.830 \\
\hline Yes & 17 & 12.1 & 14 & 11.3 & & & \\
\hline No & 123 & 87.9 & 110 & 88.7 & & & \\
\hline $\begin{array}{l}\text { Cerebrovascular } \\
\text { diseases }\end{array}$ & & & & & 1.42 & $0.63-3.21$ & 0.388 \\
\hline Yes & 17 & 12.1 & 11 & 8.9 & & & \\
\hline No & 123 & 87.9 & 113 & 91.1 & & & \\
\hline Smoking & & & & & 0.73 & $0.31-1.71$ & 0.459 \\
\hline Yes & 11 & 7.8 & 13 & 10.5 & & & \\
\hline No & 129 & 92.2 & 111 & 89.5 & & & \\
\hline Aneurysm treatment & & & & & 0.27 & $0.13-0.58$ & $<0.001$ \\
\hline Yes & 11 & 17.9 & 28 & 12.8 & & & \\
\hline No & 129 & 92.1 & 90 & 77.1 & & & \\
\hline
\end{tabular}

GCS: Glasgow Coma Scale; MAP: mean arterial pressure

\section{DISCUSSION}

In this study, we found that the case fatality rate of aSAH was $53.1 \%$. This result is considered very high compared to developed countries such as Australia and New Zealand $(27.2 \%),{ }^{(26)}$ the Netherlands $(30.0 \%),{ }^{(25)}$ England $(24.3 \%),{ }^{(24)}$ and Canada (21.5\%) ${ }^{(27)}$ and still higher compared to other studies in developing countries such as India $(38.5 \%){ }^{(28)}$ and Iran $(44.2 \%){ }^{(29)}$

Our study shows that subjects with older age were less likely to survive. Similar results have been found in other studies, in which higher age also correlates with higher risk of death..$^{(30,31)}$ A recently conducted study by Kanamaru et al. ${ }^{(32)}$ showed that older patients had more comorbidities, presented with poor World

Table 2. Multivariate logistic regression model

\begin{tabular}{lccc}
\hline & aOR & $\mathbf{9 5 \%}$ CI & p-value \\
\hline Age $<45$ years & 0.39 & $0.20-0.76$ & 0.006 \\
MAP $\geq 135 \mathrm{mmHg}$ & 2.29 & $1.10-4.39$ & 0.025 \\
Aneurysm treatment & 0.34 & $0.16-0.74$ & 0.006 \\
\hline
\end{tabular}

aOR: adjusted odds ratio 


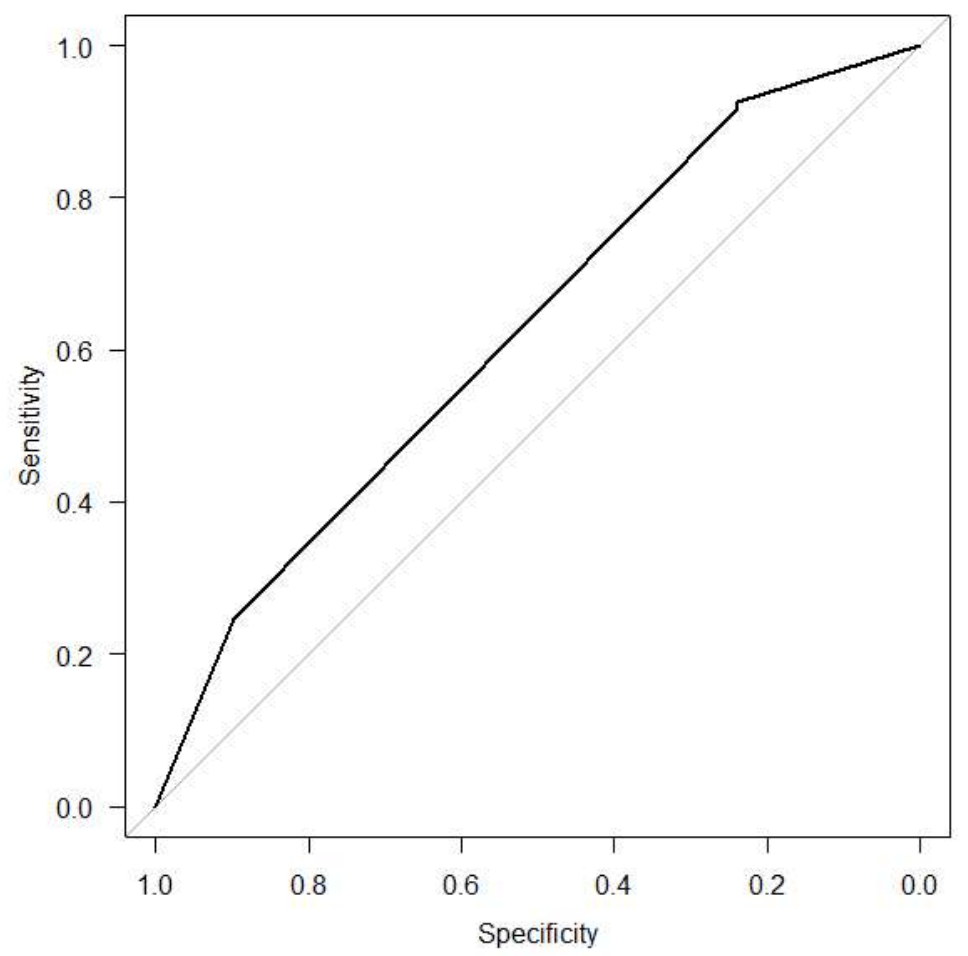

Figure 1. Receiver operating characteristics graph of the final prediction mode

Federation of Neurological Societies (WFNS) grades, that resulted in poor outcomes. An interesting study in the Netherlands showed that from 1997 to 2006 there was a decline of $1.6 \%$ in the yearly mortality rate of aneurysmal SAH due to improvements in health facilities, but not in the older age group, in which the mortality rate remained constant. ${ }^{(33)}$ Even in a group of patients that underwent aneurysm clipping, older patients still had worse outcomes compared to younger ones. ${ }^{(34)}$ However, not all studies had similar results. A study by Yue et al. ${ }^{(35)}$ showed that age was not associated with prognosis in aSAH patients who underwent endovascular coiling.

A low initial degree of consciousness measured using the GCS score on admission was found to be associated with a higher risk of mortality. This result is in line with a study in Uruguay where patients with the lowest GCS score of 3 had the highest mortality rate ( $82 \%)$ that was significantly different with the higher GCS scores. ${ }^{(36)}$ A study in the USA also showed that $77.5 \%$ of deaths occurred in the group of patients with a GCS of 3 to $8 .^{(37)}$ Loss of consciousness at onset was found to be one of the risk factors of poor outcome in aSAH. ${ }^{(38)}$ The degree of consciousness that is measured with the GCS score is commonly used as a predictor of outcomes in patients with both traumatic and non-traumatic brain injuries. ${ }^{(39)}$

In the present study, the higher mean arterial pressure (MAP) that was measured was found to be associated with higher risk of death. There were not many studies that we could find which used MAP as a prognostic factor. Zafar et al. ${ }^{(40)}$ stated that maximum MAP measured within the first three admission days predicted outcome. Other studies used the systolic blood pressure (SBP) as a prognostic factor. A study in Finland showed that an increase of 21.4 $\mathrm{mmHg}$ in systolic blood pressure could contribute to a higher risk of sudden death caused by SAH (RH 1.34; CI 95\% 1.091.65). ${ }^{(41)}$ Rodriguez et al. ${ }^{(42)}$ also showed that patients who had a maximum systolic blood pressure of more than $160 \mathrm{mmHg}$ within the first four hours of admission had worse outcomes. 
A survey of members of the Neurocritical Care Society (NCS) showed that more neurointensivists (neurology and non-neurology) preferred to use SBP as a target of aneurysm treatment in aSAH compared to MAP. ${ }^{(43)}$ However, the ability of the human auto-regulation system to increase MAP in response to increased intracranial pressure (ICP) to maintain cerebral perfusion should be taken into account, therefore MAP can also be used to monitor the patient's condition. Patients with effective autoregulation had a higher chance of good outcome. ${ }^{(44)}$ The pressure reactivity index (PRx) is sometimes used to monitor patients' autoregulation. This measurement calculates the correlation of MAP fluctuation with changes in ICP, which might be more important in the initial phase of aSAH. A study in a neurological intensive care unit (NICU) in Austria showed that a higher PRx value was associated with the occurrence of delayed cerebral ischemia. ${ }^{(45)}$ Treatment of high blood pressure in patients with SAH has to be done carefully to prevent secondary ischemia. ${ }^{(46,47)}$

Aneurysm treatment was found to be a protective factor against mortality in aneurysmal SAH. The number of aneurysm treatment procedures done in our hospital was as low as $14.8 \%$ of all aneurysmal SAH patients, with most of the procedures $(92.3 \%)$ being endovascular coiling. Re-bleeding is one of the contributors of death in aneurysmal SAH, which can be prevented by aneurysm treatment. A study in the Netherlands showed that the decrease in deaths caused by re-bleeding (aRR 0.68 ; CI 95\% 0.52-0.90) paralleled the shift of earlier aneurysm treatment from day-4 after SAH to day-1 after SAH. ${ }^{(25)}$ The 1-year mortality rate of untreated ruptured aneurysms in Helsinki (Finland) was as high as $65 \%$, varying with admission delay and clinical status. ${ }^{(48)}$ Aneurysm treatment does not only decrease patients' mortality, since a US study showed that early aneurysm treatment also reduced hospitalization cost. ${ }^{(49)}$ These studies showed that endovascular neurointervention facilities are essential to decrease mortality rate in aneurysmal SAH. Treatment in high-volume centers have been proved to increase functional outcome and decrease mortality, whereas delayed transport to neurosurgical facilities was also associated with increased mortality. ${ }^{(50,51)}$ This data can be used as reference to encourage the families of patients that are to be referred to endovascular neurointervention facilities.

We did multivariate analysis on 3 variables, namely age, mean arterial pressure and aneurysm treatment, and this model proved to be statistically significant. However, its area under ROC was 0.63 , signifying that the combination of these three variables was incapable of predicting mortality in aneurysmal SAH patients. One way to make a better model for predicting mortality in aneurysmal SAH patients would be the addition of the other variables used in previous studies, such as aneurysm characteristics including aneurysm location, size and type; patients' clinical scores such as the WFNS score or the Hunt-Hess score on admission; and occurrence of rebleeding, delayed cerebral ischemia and cerebral vasospasm.

There are a number of limitations of our study. This study was a retrospective analysis of archived data. During data collection, we could not find all medical records that were listed as subarachnoid hemorrhage in the register. Moreover, the severity of aneurysmal SAH in individual cases was not recorded. Better medical record management is suggested for the medical records center in Dr. Soetomo General Hospital.

The findings in this study have clinical implications in terms of predicting patients' outcome at the time of admission and of supporting clinical decisions of physicians whether or not to administer maximum treatment. This information is also needed by the families to know the possible outcomes of patients' current conditions. The results showing aneurysm treatment to be a strong independent protective factor could be used to encourage 
stable patients with a good prognosis to be referred to a higher neurosurgical unit for aneurysm treatment despite Indonesia's geographical challenges.

This study raised the question on how MAP as a blood pressure parameter could affect patients' outcome. Further studies on this matter should be done to evaluate the prognostic values of various blood pressure parameters. However, as stated before, the final logistic regression model in this study was not as accurate as expected in predicting outcome. Therefore, further research on other possible predictors should be done to develop a more accurate predictive model for aSAH mortality.

\section{CONCLUSIONS}

Patients with higher mean arterial pressure on initial measurement had higher risks of mortality. But younger age and any form of aneurysm treatment had lower risks of mortality. More endovascular neurointervention facilities are needed to decrease the mortality rate of aneurysmal SAH.

\section{CONFLICT OF INTEREST}

The authors have no conflicts of interest to declare.

\section{ACKNOWLEDGEMENT}

We thank Dr. Budi Utomo, dr., M.Kes. and J. Eko Wahono, dr., Sp.S, M.Kes. for suggestions of the method and statistical analysis used in this study.

\section{CONTRIBUTORS}

NNR and AFD built the database and did the data collection. NNR did all the analysis. AFS supervised the study and revised the article. All authors have read and approved the final manuscript.

\section{REFERENCES}

1. Catapano J, Lawton MT. Subarachnoid hemorrhage. In: Joaquim AF, Ghizoni E, Tedeschi $\mathrm{H}$, Ferreira MAT, editors. Fundamentals of neurosurgery: a guide for clinicians and medical students. Cham (Switzerland): Springer;2019. p:111-27. doi: 10.1007/978-3-030-17649-5 8.

2. Korja M, Lehto H, Juvela S,Kaprio J. Incidence of subarachnoid hemorrhage is decreasing together with decreasing smoking rates. Neurology 2016;87:1118-23. doi: 10.1212/WNL.000000000000 3091.

3. Okon M, Adebobola N, Julius S, et al. Stroke incidence and case fatality rate in an urban population. J Stroke Cerebrovasc Dis 2015;24:7717. doi: 10.1016/j.jstrokecerebrovasdis.2014.11.004.

4. Mackey J, Khoury J, Alwell K, et al. Stable incidence but declining case-fatality rates of subarachnoid hemorrhage in a population. Neurology 2016;87:2192-7. doi: 10.1212/WNL.0000000000003353.

5. Ikawa F, Morita A, Nakayama T, et al. A registerbased SAH study in Japan: high incidence rate and recent decline trend based on lifestyle. $J$ Neurosurg 2020;27:1-9. doi: 10.3171/2020.1. jns192848.

6. Nabaweesi-Batuka J, Kitunguu PK, Kiboi JG. Pattern of cerebral aneurysms in a Kenyan population as seen at an urban hospital. World Neurosurg 2016;87:255-65. doi: 10.1016/j.wneu. 2015.09.061.

7. Wáng YXI, He J, Zhang L, et al. A higher aneurysmal subarachnoid hemorrhage incidence in women prior to menopause: a retrospective analysis of 4,895 cases from eight hospitals in China. Quant Imaging Med Surg 2016;6:151-6. doi: 10.21037/qims.2016.01.06.

8. Brown R, Broderick J. Unruptured intracranial aneurysms: epidemiology, natural history, management options, and familial screening. Lancet Neurol 2014;13:393-404. doi: 10.1016/ S1474-4422(14)70015-8.

9. Etminan N, Chang H, Hackenberg K, et al. Worldwide incidence of aneurysmal subarachnoid hemorrhage according to region, time period, blood pressure, and smoking prevalence in the population. JAMA Neurol 2019; 76:588. doi: 10.1038/nrneurol.2016.150.

10. Kurtelius A, Kurki M, von und zu Fraunberg M, et al. Saccular intracranial aneurysms in children when both parents are sporadic or familial carriers of saccular intracranial aneurysms. Neuroepidemiology 2018;52:47-54. doi: 10.1159/ 000493856. 
11. Chan D, Abrigo J, Cheung T, et al. Screening for intracranial aneurysms? Prevalence of unruptured intracranial aneurysms in Hong Kong Chinese. J Neurosurg 2016;124:1245-9. doi: 10.3171/2015.4. JNS142938.

12. Galea J, Dulhanty L, Patel H. Predictors of outcome in aneurysmal subarachnoid hemorrhage patients. Stroke 2017;48:2958-63. doi: 10.1161/ STROKEAHA.117.017777.

13. Stienen M, Germans M, Burkhardt J, et al. Predictors of in-hospital death after aneurysmal subarachnoid hemorrhage. Stroke 2018;49:333-40. doi: 10.1161/STROKEAHA.117.019328.

14. Schuss P, Hadjiathanasiou A, Borger V, Wispel C, Vatter H, Güresir E. Poor-grade aneurysmal subarachnoid hemorrhage: factors influencing functional outcome-a single-center series. World Neurosurg 2016;85:125-9. doi: 10.1016/j.wneu.2015.08.046.

15. Goldberg J, Schoeni D, Mordasini P, et al. Survival and outcome after poor-grade aneurysmal subarachnoid hemorrhage in elderly patients. Stroke 2018;49:2883-9. doi: 10.1161/ STROKEAHA.118.022869.

16. Witsch J, Frey H, Patel S, et al. Prognostication of long-term outcomes after subarachnoid hemorrhage: the FRESH score. Ann Neurol 2016;80:46-58. doi: 10.1002/ana.24675.

17. Shimamura N, Naraoka M, Katagai T, et al. Analysis of factors that influence long-term independent living for elderly subarachnoid hemorrhage patients. World Neurosurg 2016;90:504-20. doi: 10.1016/j.wneu.2016.03.057.

18. Dasenbrock H, Rudy R, Smith, T, et al. Hospitalacquired infections after aneurysmal subarachnoid hemorrhage: a nationwide analysis. World Neurosurg 2016;88:459-74. doi: 10.1016/j.wneu.2015.10.054.

19. Foreman P, Chua M, Harrigan M, et al. Association of nosocomial infections with delayed cerebral ischemia in aneurysmal subarachnoid hemorrhage. J Neurosurg 2016;125:1383-9. doi: 10.3171/2015.10.JNS151959.

20. Wan A, Jaja B, Schweizer T, Macdonald R. Clinical characteristics and outcome of aneurysmal subarachnoid hemorrhage with intracerebral hematoma. J Neurosurg 2016;125:1344-51. doi: 10.3171/2015.10.JNS151036.

21. Chugh C, Nyirjesy S, Nawalinski K, et al. Red blood cell distribution width is associated with poor clinical outcome after subarachnoid hemorrhage: a pilot study. Neurocrit Care 2015;23:217-24. doi: 10.1007/s12028-015-0117-x.

22. Al-Mufti F, Misiolek K. Roh D, et al. White blood cell count improves prediction of delayed cerebral ischemia following aneurysmal subarachnoid hemorrhage. Neurosurgery 2019;84:397-403. doi: 10.1093/neuros/nyy045.

23. Ahn S, Savarraj J, Pervez M, et al. The subarachnoid hemorrhage early brain edema score predicts delayed cerebral ischemia and clinical outcomes. Neurosurgery 2017;83:137-45. doi: 10.1093/neuros/nyx364.

24. Mukhtar T, Molyneux A, Hall N, et al. The falling rates of hospital admission, case fatality, and population-based mortality for subarachnoid hemorrhage in England, 1999-2010. J Neurosurg 2016;125:698-704. doi: 10.3171/2015.5.JNS142115.

25. Vergouwen MDI, Fa AVJT, Algra A, Rinkel G. Time trends in causes of death after aneurysmal subarachnoid hemorrhage. Neurology 2015;86:5963. doi: 10.1212/WNL.0000000000002239.

26. Udy AA, Vladic C, Saxby ER, et al. Subarachnoid hemorrhage patients admitted to intensive care in Australia and New Zealand. Crit Care Med 2017; 45:e138-e45. doi: 10.1097/CCM.0000000000002059.

27. Chan V, Lindsay P, McQuiggan J, Zagorski B, Hill M, O'Kelly C. Declining admission and mortality rates for subarachnoid hemorrhage in Canada between 2004 and 2015. Stroke 2019;50:181-4. doi: 10.1161/STROKEAHA.118.022332.

28. Sodhi H, Savardekar A, Mohindra S, Chhabra R, Gupta V, Gupta S. The clinical profile, management, and overall outcome of aneurysmal subarachnoid hemorrhage at the neurosurgical unit of a tertiary care center in India. J Neurosci Rural Pract 2014;5:118-26. doi: 10.4103/0976-3147.131650.

29. Rezvani M, Ghandehari K, Ahmadi F, Afzalnia A. Results of surgical and non-surgical treatment of aneurysms in Iran. ARYAAtheroscler 2011;6:1369.

30. van Donkelaar C, Bakker N, Veeger N, et al. Predictive factors for rebleeding after aneurysmal subarachnoid hemorrhage. Stroke 2015;46:21006. doi: 10.3171/2016.1.JNS152136.

31. Konczalla J, Gessler F, Bruder M, Berkefeld J, Marquardt G, Seifert V. Outcome after subarachnoid hemorrhage from blood blister-like aneurysm rupture depends on age and aneurysm morphology. World Neurosurg 2017;105:94451.e1.doi: 10.1016/j.wneu.2017.06.129

32. Kanamaru H, Kawakita F, Asada R, et al. Prognostic factors varying with age in patients with aneurysmal subarachnoid hemorrhage. J Clin Neurosci 2020;76:118-25. doi: 10.1016/j.jocn.2020. 04.022 .

33. Nieuwkamp D, Vaartjes I, Algra A, Bots M, Rinkel G. Age- and gender-specific time trend in risk of death of patients admitted with aneurysmal subarachnoid hemorrhage in the Netherlands. Int 
J Stroke 2013;8 Suppl A100:90-4. doi: 10.1111/ ijs.12006.

34. Orakdogen M, Emon ST, Somay H, Engin T, Ates $\mathrm{O}$, Berkman MZ. Prognostic factor in patients who underwent aneurysmal clipping due to spontaneous subarachnoid hemorrhage. Turk Neurosurg 2016;26:840-8. doi: 10.5137/10195149.JTN.13654-14.1.

35. Yue Q, Liu Y, Leng B, et al. A prognostic model for early post-treatment outcome of elderly patients with aneurysmal subarachnoid hemorrhage. World Neurosurg 2016;95:253-61. doi: 10.1016/ j.wneu.2016.08.020.

36. Tommasino N, Saravia M, RodriguezA, Mizraji R. Epidemiologic and evolutionary profile of patients with subarachnoid hemorrhage with Glasgow Coma Scale score of 8 or less who entered the follow-up program of the National Institute of Donation and Transplantation. Transplant Proc 2018;50:405-7. doi: 10.1016/j.transproceed.2017. 12.047.

37. Lee V, Ouyang B, John S, et al. Risk stratification for the in-hospital mortality in subarachnoid hemorrhage: the HAIR score. Neurocrit Care 2014;21:14-9. doi: 10.1007/s12028-013-9952-9.

38. Suwatcharangkoon S, Meyers E, Falo C, et al. Loss of consciousness at onset of subarachnoid hemorrhage as an important marker of early brain injury. JAMA Neurol 2016;73:28. doi: 10.1001/ jamaneurol.2015.3188.

39. Khanal K, Bhandari S, Shrestha N, Acharya S, Marhatta M. Comparison of outcome predictions by the Glasgow coma scale and the full outline of unresponsiveness score in the neurological and neurosurgical patients in the Intensive Care Unit. Indian J Crit Care Med 2016;20:473-6. doi: 10.4103/ 0972-5229.188199.

40. Zafar SF, Postma EN, Biswal S, et al. Electronic health data predict outcomes after aneurysmal subarachnoid hemorrhage. Neurocrit Care 2018;28:184-93. doi: 10.1007/s12028-017-0466-8.

41. Lindbohm J, Kaprio J, Jousilahti P, Salomaa V, Korja M. Risk factors of sudden death from subarachnoid hemorrhage. Stroke 2017;48:2399404. doi: 10.1161/STROKEAHA.117.018118.

42. Rodriguez DR, Matamoro CS, Cue F, Hernandez JLM, Sanchez YP, Nellar JP. Factors associated with poor outcome for aneurysmal subarachnoid haemorrhage in a series of 334 patients. Neurologia 2017;32:15-21. doi: 10.1016/j.nrl.2014. 12.006 .
43. Brown RJ, Kumar A, McCullough LD, Butler K. A survey of blood pressure parameters after aneurysmal subarachnoid hemorrhage. Int $\mathrm{J}$ Neurosc 2017;127:1-8. doi: 10.3109/00207454.2016. 1138952.

44. Brooks FA, Ughwanogho U, Henderson GV, BlackSchaffer R, Sorond FA, Tan CO. The link between cerebrovascular hemodynamics and rehabilitation outcomes after aneurysmal subarachnoid hemorrhage. Am J Phys Med Rehabil 2018;97:30915. doi: 10.1097/PHM.0000000000000886.

45. Gaasch M, Schiefecker AJ, Kofler M, et al. Cerebral autoregulation in the prediction of delayed cerebral ischemia and clinical outcome in poor-grade aneurysmal subarachnoid hemorrhage patients. Crit Care Med 2018;46:77480. doi: 10.1097/CCM.0000000000003016.

46. Wajngarten M, Silva GS. Hypertension and stroke: update on treatment. Eur Cardiol Rev 2019;14:111-5. doi: 10.15420/ecr.2019.11.1.

47. Connolly ES Jr, Rabinstein AA, Carhuapoma JR, et al. Guidelines for the management of aneurysmal subarachnoid hemorrhage: a guideline for healthcare professionals from the American Heart Association/American Stroke Association. Stroke 2012;43:1711-37. doi: 10.1161/STR.0b013e3182587839.

48. Korja M, Kivisaari R, Jahromi BR, Lehto H. Natural history of ruptured but untreated intracranial aneurysms. Stroke 2017;48:1081-4. doi: 10.1161/ STROKEAHA.116.015933.

49. Sonig A, Shallwani H, Natarajan S, et al. Better outcomes and reduced hospitalization cost are associated with ultra-early treatment of ruptured intracranial aneurysms: a US Nationwide Data Sample Study. Neurosurgery 2018;82:497-505. doi: 10.1093/neuros/nyx241.

50. Rush B, Romano K, Ashkanani M, McDermid R, Celi L. Impact of hospital case-volume on subarachnoid hemorrhage outcomes: a nationwide analysis adjusting for hemorrhage severity. J Crit Care 2017;37:240-3. doi: 10.1016/ j.jcrc.2016.09.009.

51. van Lieshout JH, Bruland I, Fischer I, et al. Increased mortality of patients with aneurysmatic subarachnoid hemorrhage caused by prolonged transport time to a high-volume neurosurgical unit. Am J Emerg Med 2017;35:45-50. doi: 10.1016/ j.ajem.2016.09.067. 